\title{
Identification of a Maize Kernel Stress-Related Protein and Its Effect on Aflatoxin Accumulation
}

\author{
Z.-Y. Chen, R. L. Brown, K. E. Damann, and T. E. Cleveland
}

First and third authors: Department of Plant Pathology and Crop Physiology, Louisiana State University Agricultural Center, Baton Rouge 70803; and second and fourth authors: Southern Regional Research Center, U.S. Department of Agriculture-Agricultural Research Service, New Orleans, LA 70179.

Accepted for publication 14 April 2004.

\begin{abstract}
Chen, Z.-Y., Brown, R. L., Damann, K. E., and Cleveland, T. E. 2004. Identification of a maize kernel stress-related protein and its effect on aflatoxin accumulation. Phytopathology 94:938-945.

Aflatoxins are carcinogens produced mainly by Aspergillus flavus during infection of susceptible crops such as maize. Through proteomic comparisons of maize kernel embryo proteins of resistant and susceptible genotypes, several protein spots previously were found to be unique or upregulated in resistant embryos. In the present study, one of these protein spots was sequenced and identified as glyoxalase I (GLX-I; EC 4.4.1.5). The full-length cDNA of the glyoxalase I gene ( $g l x-I)$ was cloned. GLX-I constitutive activity was found to be significantly higher in the resistant maize lines compared with susceptible ones. After kernel in-
\end{abstract}

ABSTRACT fection by A. flavus, GLX-I activity remained lower in susceptible genotypes than in resistant genotypes. However, fungal infection significantly increased methylglyoxal (MG) levels in two of three susceptible genotypes. Further, MG was found to induce aflatoxin production in A. flavus culture at a concentration as low as $5.0 \mu \mathrm{M}$. The mode of action of MG may be to stimulate the expression of aflR, an aflatoxin biosynthesis regulatory gene, which was found to be significantly upregulated in the presence of 5 to $20 \mu \mathrm{M}$ MG. These data suggest that GLX-I may play an important role in controlling MG levels inside kernels, thereby contributing to the lower levels of aflatoxins found in resistant maize genotypes.

Additional keywords: host resistance, real-time PCR, S-D-lactoylglutathione methylglyoxal lyase.
Infection of maize (Zea mays L.) by Aspergillus flavus Link:Fr. and the subsequent accumulation of the toxic and highly carcinogenic secondary metabolites, aflatoxins, is a serious agricultural problem, especially in maize grown under drought conditions $(19,31)$. Aflatoxin contamination significantly reduces the value of grain both as an animal feed and as an export commodity (29). It also poses health hazards to humans $(24,31)$ and domestic animals (29).

During the past two decades, maize genotypes with natural preharvest resistance to aflatoxin production have been identified through field screening $(11,33,39)$. The poor agronomic quality of these lines renders them of little direct commercial value (7). The lack of identified resistance-associated traits in these genotypes has slowed the incorporation of resistance into lines with commercially acceptable genetic backgrounds.

Maize kernel protein expression has been implicated in resistance to A. flavus infection and aflatoxin production $(7,14,23,27)$. Two-dimensional polyacrylamide gel electrophoresis (2-D PAGE) profiles of embryo proteins of two resistant maize genotypes were recently compared with those of five susceptible genotypes (13). One such protein, denoted as spot \#1175, was expressed at higher levels (fivefold) in resistant genotypes Mp420 and Mp313E than in susceptible genotypes, some of which appeared to completely lack the protein (Fig. 1).

In the current study, protein spot \#1175 from resistant genotype Mp420 was sequenced and identified as glyoxalase I (GLX-I) ( $S$ D-lactoylglutathione methylglyoxal lyase; EC 4.4.1.5). This en-

Corresponding author: R. L. Brown; E-mail address: rbrown@srrc.ars.usda.gov

Publication no. P-2004-0628-02R

This article is in the public domain and not copyrightable. It may be freely reprinted with customary crediting of the source. The American Phytopathological Society, 2004. zyme, along with glyoxalase II (GLX-II) (EC 3.1.2.6), is involved in the conversion of cytotoxic methylglyoxal (MG) into D-lactate (18) (Fig. 2). A recent study by Veena et al. (36) suggested an important role for GLX-I in conferring tolerance to transgenic tobacco plants grown under stress conditions (methylglyoxal or high salt).

The objective of the present study was to investigate the potential for direct involvement of GLX-I in host resistance against aflatoxigenic fungi. Here, we cloned the GLX-I cDNA based on partial amino acid sequences, assayed its enzyme activities in aflatoxin-resistant and -susceptible maize kernels, both fungal-infected and noninfected, and investigated the effect of aflatoxin on GLX-I activity. We also assessed the effect of methylglyoxal, GLX-I's substrate, on aflatoxin production and the expression of aflatoxin pathway genes in liquid culture. Higher GLX-I activities observed in kernels of resistant lines with or without A. flavus infection, and the effect of MG on the expression of aflatoxin pathway genes and on aflatoxin production, suggest a direct role for GLX-I in host resistance against aflatoxin accumulation.

\section{MATERIALS AND METHODS}

Chemicals and materials. Chemicals for 2-D protein gels and kernels of resistant and susceptible maize genotypes were obtained from the same source as in a previous study (13). Resistance or susceptibility of genotypes to aflatoxin accumulation was determined by breeders in field trials repeated over different environments and times using artificial and natural infection. Aflatoxin standards were purchased from Sigma-Aldrich (St. Louis) and prepared according the manufacturer's instructions.

2-D Gel electrophoresis, peptide sequencing, and database sequence homology analysis. Protein spot \#1175 identified in previous comparisons (13) was recovered from five 2-D gels and sequenced as previously described using PE SCIEX API 3000 
(Applied Biosystems, Foster City, CA) electrospray ionization tandem mass spectrometry (ESI-MS/MS) equipped with a Protana nanospray source (Protana, Odense, Denmark) (13). Peptide sequence homology searches were performed using BLAST (2) against known proteins or translated open reading frames of expressed sequence tags (ESTs) in databases at the National Center for Biotechnology Information (NCBI) and SWISS-Prot.

Cloning of the full-length GLX-I gene $(g l x-I)$ from a maize embryo cDNA library. Two degenerate primers were made, 5'CTTCCAICC(G/A)TCIGG(G/A)TC-3' (GLX-R1) and 5'-AG(G/A)AA(G/A)TCIGT(G/A)TT(G/A)TC(G/C/A)AC-3' (GLX-R2), based on peptide sequences from peaks $\mathrm{b}$ and $\mathrm{c}$, respectively (Fig. 3 ). The first round of polymerase chain reaction (PCR) to amplify the $5^{\prime}$ end of $g l x-I$ cDNA was performed in $50 \mu \mathrm{l}$ using a M13(-20) and GLX-R2 primer pair and $1 \mu \mathrm{l}$ of undiluted cDNA library (titer $1.1 \times 10^{7}$ plaque forming units per $\mathrm{ml}$ ) as template (provided by T. Rocheford, Department of Crop Sciences, University of Illinois, Urbana). The PCR conditions consisted of $95^{\circ} \mathrm{C}$ for $2 \mathrm{~min}$, followed by two cycles of $95^{\circ} \mathrm{C}$ for $50 \mathrm{~s}, 50^{\circ} \mathrm{C}$ for $3 \mathrm{~min}$, and $72^{\circ} \mathrm{C}$ for $1.5 \mathrm{~min}$; then followed by 30 cycles of $95^{\circ} \mathrm{C}$ for $50 \mathrm{~s}, 53^{\circ} \mathrm{C}$ for $1 \mathrm{~min}$, and $72^{\circ} \mathrm{C}$ for $1.5 \mathrm{~min}$; and a final extension of $72^{\circ} \mathrm{C}$ for $15 \mathrm{~min}$. The second round of PCR was performed using primers M13(-20) and GLX-R1 and $1 \mu \mathrm{l}$ of 1:20 diluted first-round PCR product as template. The PCR conditions were similar to the first round except the annealing temperature was $53^{\circ} \mathrm{C}$ throughout. The $1.0-\mathrm{kb}$ PCR product was cloned into the pCR2.1-TOPO vector for sequencing according to manufacturer's instructions (Invitrogen, Carlsbad, CA). Primers GLXEND-03 (5'-CTGTTATTGCCTTCGCACA-3', made based on the cloned $g l x-I 5^{\prime}$ end sequence) and M13rev were used to clone the $3^{\prime}$ end of the $g l x-I$ from the same cDNA library. PCR conditions were the same as the first round of PCR for the $g l x-I 5^{\prime}$-end cDNA described above, except that the annealing temperature for the first two cycles was $45^{\circ} \mathrm{C}$, followed by $50^{\circ} \mathrm{C}$ for the remaining cycles. The second round of PCR for the glx-I 3'-end cDNA was performed similarly using primer GLXEND-05 (5'-TGTTGGTGACCTTGAGCGTTC- $3^{\prime}$ ), which also was made based on the cloned glx-I 5'-end cDNA sequence, and M13rev and $1 \mu l$ of the 1:20 diluted first-round PCR mixture as template. PCR conditions were similar to the first round, except the annealing temperature was $53^{\circ} \mathrm{C}$. The $0.7-\mathrm{kb}$ PCR product then was cloned as above and sequenced using a dye termination reaction on an ABI 377 DNA sequencer (Applied Biosystems Inc., Warrington, UK).

GLX-I activity assays in resistant and susceptible kernel embryos. GLX-I enzyme activity in kernel embryo protein extracts of 11 maize genotypes (6 resistant and 5 susceptible) was measured according to Racker (32) and Johansen et al. (26). The assay mixture contained $100 \mathrm{mM}$ sodium phosphate buffer, $\mathrm{pH}$ 7.5, 3.5 mM MG, $1.7 \mathrm{mM}$ reduced glutathione $(\mathrm{GSH})$, and $16.0 \mathrm{mM}$ magnesium sulfate in a final volume of $1 \mathrm{ml}$. The mixture was transferred to a quartz cuvettes and incubated at $25^{\circ} \mathrm{C}$. The reaction was initiated by adding the enzyme $(0.02 \mathrm{ml}$ of extract, or boiled extract as a control), and the formation of $S$-Dlactoylglutathione thio-ester was monitored by measuring the increase in absorbance at $240 \mathrm{~nm}$ in a spectrophotometer UV1601 (Shimadzu, Columbia, MD) for $20 \mathrm{~min}$. The UV absorbance was converted to mole quantity by using the molecular coefficient of 3,370 for $S$-lactoylglutathione. One unit is defined as $1 \mu \mathrm{mol} S$ lactoylglutathione/min. The protein concentrations were determined using the Bio-Rad protein assay kit according to Bradford (6). The experiment was conducted three times.

GLX-I activity, MG content, and aflatoxin production in infected or noninfected kernels. The enzyme activity and $\mathrm{MG}$ content were assayed in total kernel protein extracts from three resistant and three susceptible genotypes with or without fungal infection along with aflatoxin quantitation. Kernels (120 per

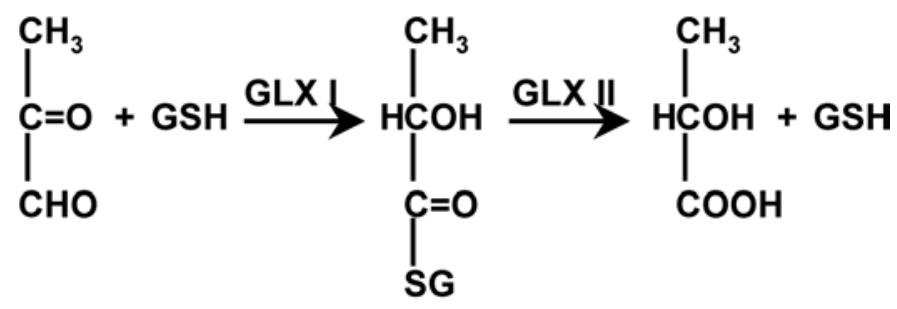

\section{Methylglyoxal S-D-lactoylglutathione D-lactate}

Fig. 2. Enzymatic conversion of methylglyoxal to D-lactate by glyoxalase I (GLX-I) and glyoxalase II (GLX-II). GLX-I catalyzes the formation of $S$-Dlactoylglutathione from methylglyoxal in the presence of reduced glutathione (GSH). S-D-lactoylglutathione is further metabolized to D-lactate and GSH by GLX II.

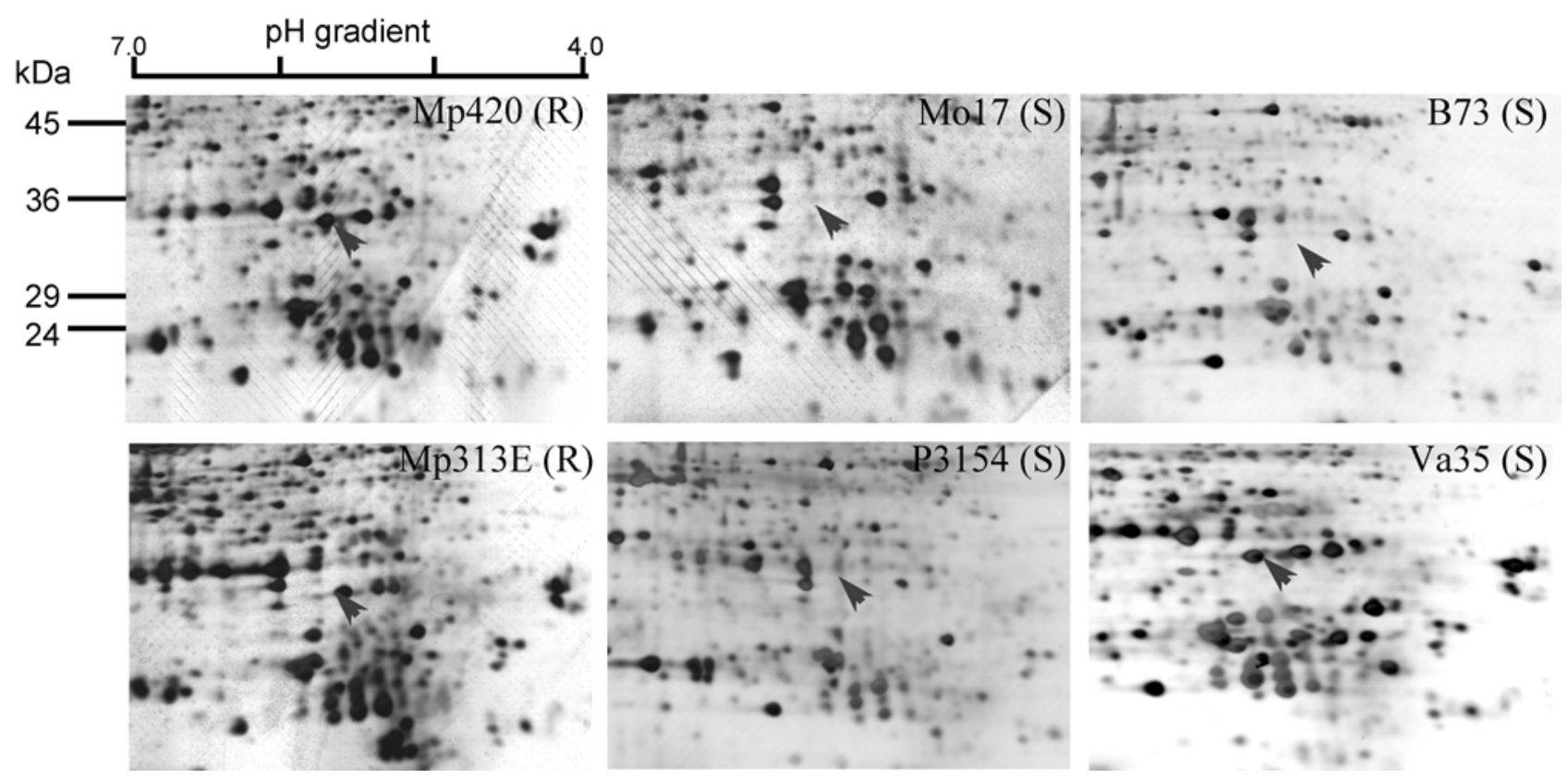

Fig. 1. Comparison of embryo proteins between resistant (R) and susceptible (S) maize genotypes using large-format two-dimensional gels. Arrows indicate the position of spot \#1175 in different genotypes. 
genotype) of three resistant (Mp420, GT-MAS:gk [MAS], and T115) and three susceptible genotypes (B73, G4666, and P3165) were surface sterilized as described (34). Eighty kernels from each genotype were inoculated with an A. flavus conidia suspension $\left(5 \times 10^{6}\right.$ conidia/ml $)$ and incubated at $31^{\circ} \mathrm{C}$ under $100 \%$ humidity for 7 days as previously described (9). At the end of incubation, 40 kernels were ground as described in a previous study (14) and the protein extract was used for GLX-I activity assay (above). The other 40 kernels (4 kernels/replicate) were bagged, dried, and used for aflatoxin quantitation using the method of Brown et al. (9). The remaining 40 kernels from each genotype were inoculated with sterile water to serve as a noninfected control and were incubated for 7 days under the same conditions before grinding for GLX-I activity assay. The experiment was conducted twice.

MG content in kernel protein extract was measured essentially as described above for the GLX-I activity assay, except that protein extracts were used as a source of MG in the GLX-I reaction. The assay mixture contained $100 \mathrm{mM}$ sodium phosphate buffer, $\mathrm{pH}$ 7.5, $1.7 \mathrm{mM}$ reduced GSH, $16.0 \mathrm{mM}$ magnesium sulfate, and 20 units of GLX-I (Sigma-Aldrich) in a final volume of $1 \mathrm{ml}$. The reaction was started by adding $20 \mu \mathrm{l}$ of various known concentrations of MG solution $(0,2,4,8,10,16$, and $20 \mu \mathrm{M})$ or protein extracts and the changes in absorbance at $240 \mathrm{~nm}$ were monitored using a Shimadzu spectrophotometer UV-1601 for $20 \mathrm{~min}$. The MG concentration in protein extract was calculated based on the standard curve between MG concentration and changes in absorbance per minute.

Inhibition of glyoxalase activity by aflatoxin. The GLX-I activity assay reaction mixture containing $100 \mathrm{mM}$ sodium phos- phate buffer, $\mathrm{pH}$ 7.5, $1.7 \mathrm{mM}$ reduced GSH, and $16.0 \mathrm{mM}$ magnesium sulfate was set up as described above. Then, different amounts of aflatoxins ( 0 to $600 \mathrm{ng}$ of $\mathrm{B}_{1}$ in $4 \mu$ l of acetone) were added to the GLX-I assay mixture. The mixture (final volume $1 \mathrm{ml}$ ) was incubated at room temperature for $5 \mathrm{~min}$ prior to the addition of MG to start the reaction. The reaction mixture containing $4 \mu \mathrm{l}$ of acetone only also was used as a control. Reduction in enzyme activity compared with the acetone-only control is expressed as the inhibition effect of aflatoxin. The experiment was conducted twice, with three replicates for each treatment.

Aflatoxin production and fungal growth of $A$. flavus in culture containing MG. A \& M medium (100 ml/flask) (1), containing various concentrations of $\mathrm{MG}(0,5,10,20,50,100,250$, and $500 \mu \mathrm{M} ; 1 \mu \mathrm{M}=0.072 \mu \mathrm{g} / \mathrm{ml})$, was inoculated with $1 \times 10^{5}$ freshly prepared $A$. flavus conidia per flask. The flasks were incubated at $31^{\circ} \mathrm{C}$ with continuous shaking (200 rpm) for 4 days. At the end of incubation, aflatoxins were extracted from the medium and mycelia using acetone and chloroform, and quantified as previously described (16). Fungal mycelium then was collected through filtration and the final weight was recorded after drying overnight at $50^{\circ} \mathrm{C}$. This experiment was conducted twice with three replicates per treatment.

Real-time reverse-transcription PCR. Real-time reverse-transcription (RT)-PCR was used to examine whether the presence of MG in fungal growth medium affected the expression of aflatoxin biosynthesis pathway genes. Fungal cultures were started as described above and MG was added at the time of inoculation. The MG concentrations used for this study were: $0,5,20,50$, and $250 \mu \mathrm{M}$. After 4 days of continuous culture at $31^{\circ} \mathrm{C}$, fungal mycelia were harvested and ground in liquid nitrogen. Total fungal

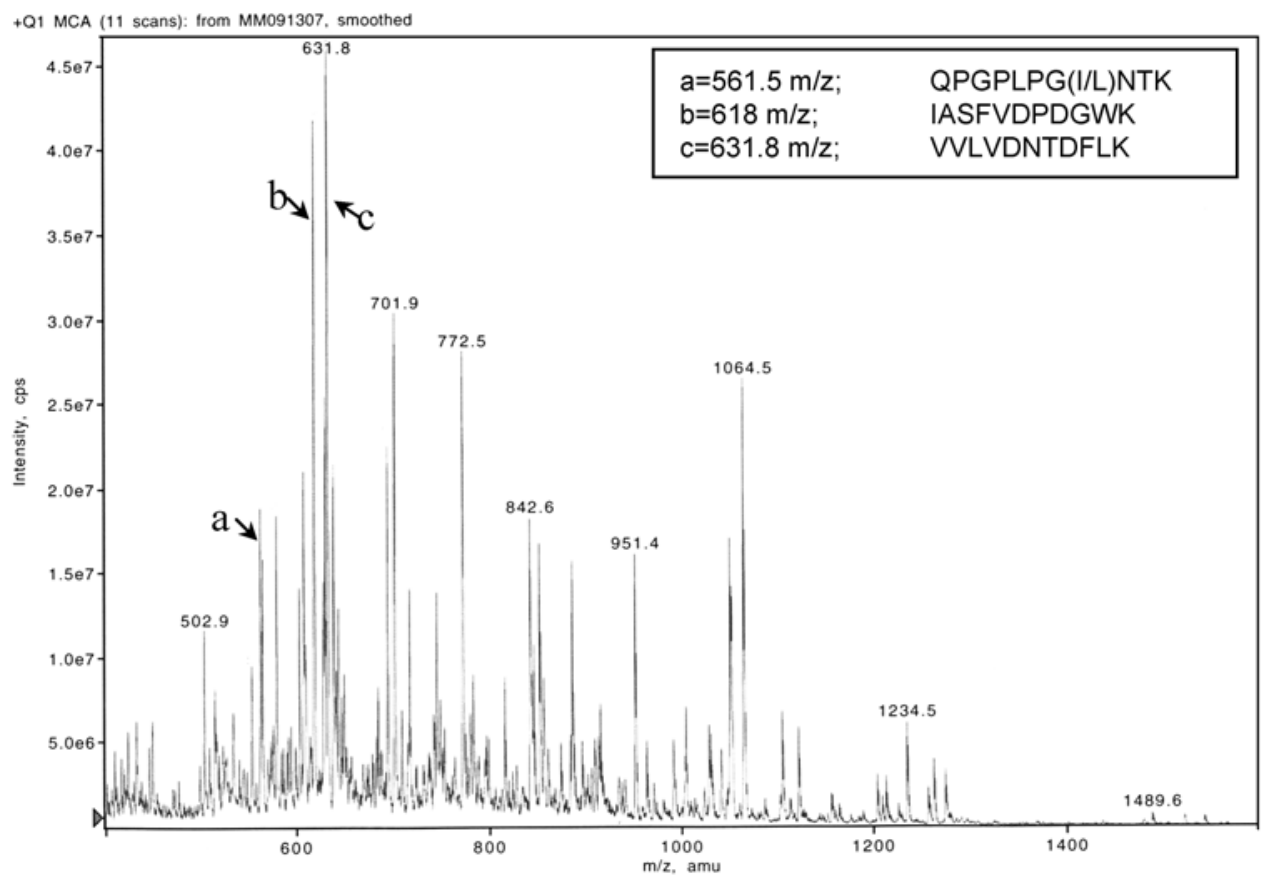

Fig. 3. Electrospray ionization peptide mass spectrum of a peptide mixture from in-gel tryptic digestion of protein spot \#1175. Peptide sequences corresponding to peaks $\mathrm{a}, \mathrm{b}$, and $\mathrm{c}$ were shown in the inset. The sequence shown in parentheses indicates that it could be either I or L at that position based on mass spectrometry; $\mathrm{m} / \mathrm{z}=$ mass and charge ratio.

TABLE 1. Real-time polymerase chain reaction primer pairs for aflatoxin biosynthesis genes used in the present study

\begin{tabular}{lll}
\hline Gene & \multicolumn{1}{c}{ Forward primer $\left(5^{\prime}\right.$ to $\left.3^{\prime}\right)$} & Reverse primer $\left(5^{\prime}\right.$ to $\left.3^{\prime}\right)$ \\
\hline $18 \mathrm{~S}$ & GCTCTTTTGGGTCTCGTAATTGG & CGCTATTGGAGCTGGAATTACC \\
aflR & CAACCTGATGACGACTGATATGG & TGCTGCCGCAGCATACC \\
aflJ & TCCTTACTGCAGCTAACCAATGG & CACACGGTACTGAATCTCAAATGC \\
nor 1 & CCTGAGGAGACGGTGTATTTGG & CGACCACGGTGCTTTTGG \\
ver 1 & CGGTGCGCCATTTTGG & GGTGACCGAACGATACAATTCC \\
p $s$ GCTCCAGCGGCAAAAGG & TTCCACTCTCCTCCGAAACG \\
\hline
\end{tabular}


RNA was isolated using the Qiagen RNeasy mini kit (Valencia, CA) and reverse transcribed into cDNA using TaqMan Reverse Transcription Reagents (Applied Biosystems) according to the manufacturers' instructions. Then, the level of aflatoxin biosynthesis pathway gene expression was quantified using an ABI 5700 Thermal Cycler with SYBR Green dye chemistry (Applied Biosystems). The primer pairs amplifying the following genes were used in real-time PCR: $18 \mathrm{~S}$ ribosomal RNA, aflR (an aflatoxin pathway transcription activator), aflJ (regulator), nor 1 (reductase), ver 1 (dehydrogenase), and pks (polyketide synthase) (40) (Table 1). Data were normalized to the $18 \mathrm{~S}$ ribosomal RNA levels. This experiment was conducted twice, each time with three replicates per treatment.

Statistical analysis. All data were analyzed using the analysis of variance procedure of the Statistical Analysis System (SAS Institute, Cary, NC). Aflatoxin data were log transformed prior to analysis to equalize variances. Means were separated by Duncan's multiple range test $(P \leq 0.05)$.

\section{RESULTS}

Protein sequencing and database query. Protein spot \#1175 was expressed at high levels (fivefold) in resistant genotypes Mp420 and Mp313E and at low or undetectable levels in most susceptible maize lines examined (Fig. 1). Spot \#1175 was excised from Coomassie-stained preparative 2-D gels and subjected to in-gel trypsin digestion. The digested peptides then were separated using liquid chromatography. Three of the peaks were selected for sequence analysis using ESI-MS/MS (Fig. 3). The peptide sequences obtained from MS are shown in the inset of Figure 3. Peptide sequence analysis of spot \#1175 showed that it has a high homology (94\%) to the C-terminus of the GLX-I protein from rice (Fig. 4).

Cloning and sequencing of cDNA encoding maize GLX-I. The $5^{\prime}$ end of $g l x-I$ was cloned from a cDNA library using PCR with two degenerate primers. Several independent PCR clones were sequenced and the sequence information confirmed that the cloned cDNAs are part of the gene or genes encoding maize GLX-I. The $3^{\prime}$ end of $g l x-I$ cDNA was cloned using 3 '-end rapid amplification of cDNA ends as described above. The full-length cDNA (GenBank accession no. AY241545; this study) is 1,154 bp long, with an open reading frame between 103 to 972, capable of encoding 290 amino acids. A BLAST search of the sequence before submitting to GenBank found several EST sequences (such as BG842523, BQ293544, BQ164210, and CA400243) from different tissues of maize that showed high homology (ranging from 97 to $99 \%$ ) to the $g l x-I$ reported in this study. The deduced molecular mass of the encoded protein is $32,302 \mathrm{Da}$ with a $\mathrm{pI}$ of 5.45 , which is very close to the estimated molecular mass $(33,914)$ and pI $(5.60)$ based on 2-D gels (13). The deduced amino acid sequence of $g l x-I$ from maize consists of two conserved homologous domains (Fig. 4, underlined), and it shares significant homology to GLX-I from Oryza sativa ( $88 \%$ identity) (accession no. AB017042). It also shares $\approx 75 \%$ sequence identity to GLX-I from Arabidopsis thaliana (accession no. AY087874), Brassica oleracea, (accession no. Q39366), and Triticum aestivum (accession no. AJ243528) (Fig. 4). All four belong to the two-domain long-type glyoxalase family (26) based on their size. The sequence similarity between maize and human, mice, or Escherichia coli GLX-I, however, was much lower (35 to 52\% identity).

Variation of GLX-I activity in different maize genotypes. The kernel embryo protein extracts, from which spot \#1175 was first identified, were assayed for GLX-I activity. High variation (as much as fourfold) of GLX-I activity was observed between genotypes, ranging from 0.216 for $\mathrm{Mp} 313 \mathrm{E}$ (resistant $[\mathrm{R}]$ ) to $0.045 \mu \mathrm{mol} S$-lactoylglutathione $\mathrm{mg}^{-1}$ of protein $\mathrm{min}^{-1}$ for P3154 (susceptible [S]) (Fig. 5). Out of 11 genotypes studied, the resistant embryos contained higher levels of GLX-I activity than the susceptible ones on either per milligram of protein or per gram embryo basis (data not shown), with the exception of MI82 (R) and Va35 (S) (Fig. 5). The level of GLX-I activity in MI82 was comparable to susceptible genotypes, whereas the activity in Va35 was comparable to resistant lines. This high level of GLX-I activ-

\begin{tabular}{|c|c|c|}
\hline consen & & eWpKkD.rR.LHvV \\
\hline GLX-TA & --PRATSFSSNDEAF & TWAKKDNRRLLHVV \\
\hline $\mathrm{GLX}-\mathrm{BO}$ & ------ MAENADLV & EWPKKDKRRELHVV \\
\hline GLX-AT & ----- MAEASDLL & EWPKKDNRRELHT \\
\hline GLX-OS & MASGSEAEKSPEVVL & EWPKKDKKRLLH \\
\hline GLX-ZM & MATGSEASKAAETVV & DWHKQDSKRMLH \\
\hline 1175 & --------------- & \\
\hline consen & GtGEGHEaIat. DV. & KlvE.ira.kgg \\
\hline GLX-TA & GAGEGHFGIATDDVA & KTVELIRA-KGGI \\
\hline $\mathrm{GLX}-\mathrm{BO}$ & GTGFGHEAISTQDVS & KMVEAVRA-KGGI \\
\hline GLX-AT & GTGEGHEAISTQDVS & KLVENVRA-KGGI \\
\hline GLX-OS & GAGEGHEAIATEDVY & KLAEKIKSSCCC \\
\hline GLX-ZM & GTGEGHEAIANDDVY & KLAENIKS-KGGKI \\
\hline 1175 & ---------------- & \\
\hline consen & LLRK.d.PeYkYTIa & MmGYa.Ed...V \\
\hline GLX-TA & LLRKRDNPEYKYTIA & MMGYGPEDQNAVI \\
\hline GLX-BO & LLRRIERPEYN-TIG & MMGYAEEYESIVI \\
\hline GLX-AT & LLRKIERPEYKYTIG & MMGYAEEYESIVI \\
\hline GLX-OS & LLRKKDVPDYKYTIA & MLGYADEDKTTV \\
\hline GLX-ZM & $\underline{\text { LLRKKDVPDYKYTIA }}$ & MLGYADEDKTTV \\
\hline 1175 & $\overline{----------------}$ & - \\
\hline en & IDPdGWK.VIVDN.D & FlKELe \\
\hline GLX-TA & LDPBGWKSVEVDNID & FAKELE \\
\hline $\mathrm{GLX}-\mathrm{BO}$ & LDPDGWKQVLVDNED & FLKELE \\
\hline GLX-AT & LDPDGWKTVLVDNKD & FLKELE \\
\hline GLX-OS & LDPDGWKVVLVDNAD & ELKELQ \\
\hline GLX-ZM & VDPDGWKVVLVDNTD & FLKELQ \\
\hline 1175 & VDPDGWKVVLVDNTD & FLK--- \\
\hline
\end{tabular}

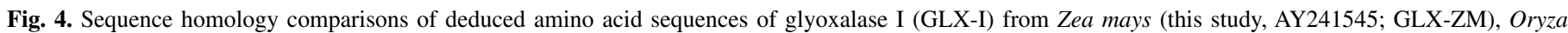

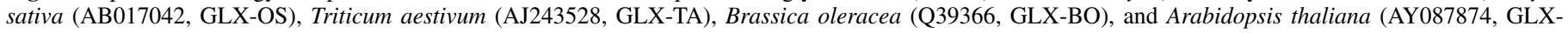

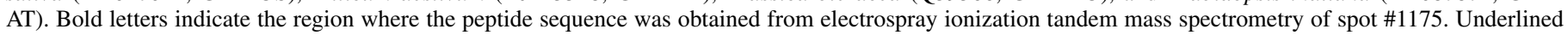

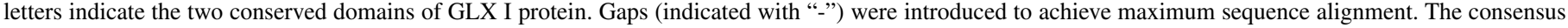

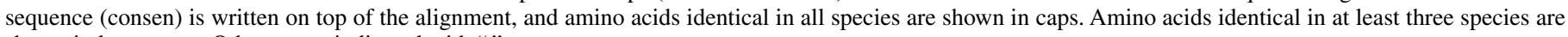
shown in lower case. Others were indicated with ".". 
ity in Va35 agrees well with its high protein level observed on 2-D gels. When the glyoxalase activity of the resistant genotypes (mean activity $=0.177 \mu \mathrm{mol} S$-lactoylglutathione $\mathrm{mg}^{-1}$ of protein $\mathrm{min}^{-1}$ ) is collectively compared with that of the susceptible genotypes $\left(0.124 \mu \mathrm{mol} S\right.$-lactoylglutathione $\mathrm{mg}^{-1}$ of protein $\left.\mathrm{min}^{-1}\right)$, the difference in mean activity between the two groups was significant (least significant difference $=0.033, P=0.05$ ).

GLX-I activity, MG content, and aflatoxin accumulation in Aspergillus flavus infected or noninfected kernels. The GLX-I activities in kernels of three resistant and three susceptible genotypes with or without Aspergillus flavus infection were determined (Table 2). In noninfected (imbibed) kernels, two resistant lines have significantly higher levels of kernel GLX-I activity than susceptible genotypes. The activity in the other resistant line (MAS) is not significantly different from the susceptible genotypes. In infected kernels, all three resistant genotypes contained significantly higher levels of GLX-I activity than susceptible ones (Table 2). Also, a significant increase in GLX-I activity was observed in resistant genotype MAS compared with the noninfected kernels. The activity did not change significantly in all other genotypes compared with the noninfected kernels (Table 2).

In noninfected kernels, the level of MG showed a pattern similar to the GLX-I activity (Table 2). Two (B73 and P3154) of the three susceptible genotypes had significantly lower levels of MG than resistant lines (Table 2). The highest MG level was measured in Mp420 (R) and the lowest level in P3154 (S). In infected kernels, MG content did not change significantly in resistant lines

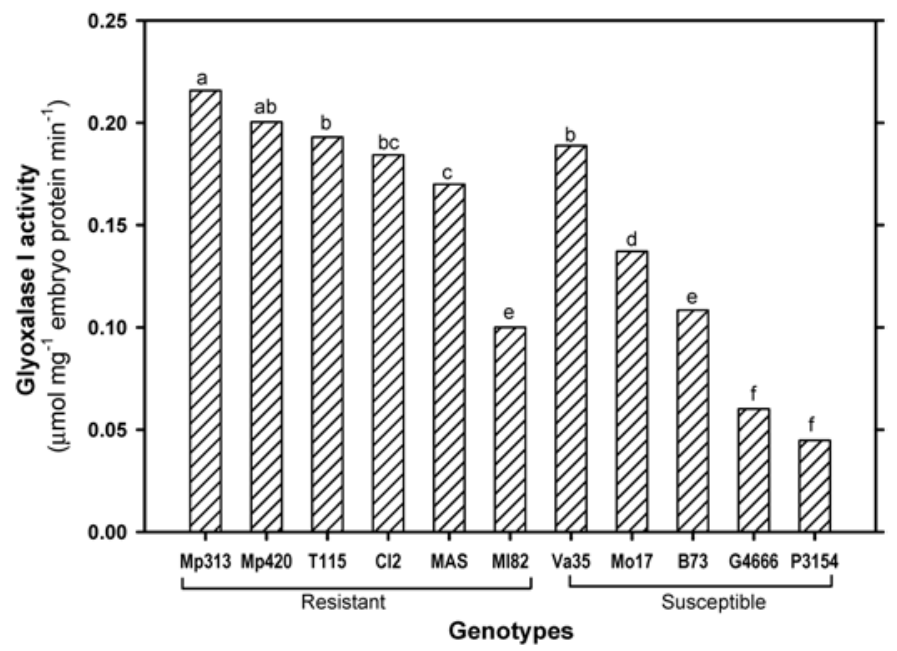

Fig. 5. Glyoxalase I activities in resistant and susceptible maize genotypes. Activity was expressed as micromoles of $S$-lactoylglutathione produced (equivalent to micromoles of methylglyoxal consumed) per minute per milligram of embryo protein. Bars labeled with the same letters are not statistically different based on Duncan's multiple range test. Resistant and susceptible genotypes are noted. compared with noninfected kernels, whereas it increased significantly in two susceptible lines, G4666 and B73 (Table 2).

Aflatoxin inhibits GLX-I activity in vitro. In mice, it has been reported that aflatoxin inhibits colon GLX-I activity and increases the sensitivity of mice to MG $(3,4)$. Here, using an in vitro assay, aflatoxin inhibited the activity of maize GLX-I as well (Fig. 6). The addition of $30 \mathrm{ng}$ of aflatoxin $\mathrm{B}_{1}$ to $1 \mathrm{ml}$ of assay mixture did not reduce activity significantly; however, in the presence of aflatoxin $B_{1}$ at 300 or $600 \mathrm{ng} / \mathrm{ml}$ of assay mixture, there was significant reduction (23.7 and $37.4 \%$, respectively) compared with the control (Fig. 6). Significant inhibition initially was seen with the addition of aflatoxin $B_{1}$ at $60 \mathrm{ng} / \mathrm{ml}$ of assay mixture (data not shown).

MG induces aflatoxin production in A. flavus culture. The effect of MG on fungal growth and aflatoxin production also was investigated. Aflatoxin production in fungal growth medium was induced by $\approx 100 \%$ in the presence of as little as $5 \mu \mathrm{M} \mathrm{MG}$ (equivalent to $0.36 \mu \mathrm{g} / \mathrm{ml}$ ) (Fig. 7). The maximum induction of aflatoxin was observed in medium containing $20 \mu \mathrm{M}$ MG. As the concentration of MG in the medium increased to above $50 \mu \mathrm{M}$, the amount of aflatoxin decreased; however, it was still significantly higher than the control. The addition of MG to fungal growth medium did not appear to inhibit fungal growth; instead, a steady increase in fungal biomass was observed with the increase in $\mathrm{MG}$ concentration in the medium (Fig. 7).

MG affects the expression of aflatoxin biosynthetic pathway genes. The expression of aflatoxin pathway genes in the presence of MG also was examined using real-time PCR. The data pre-

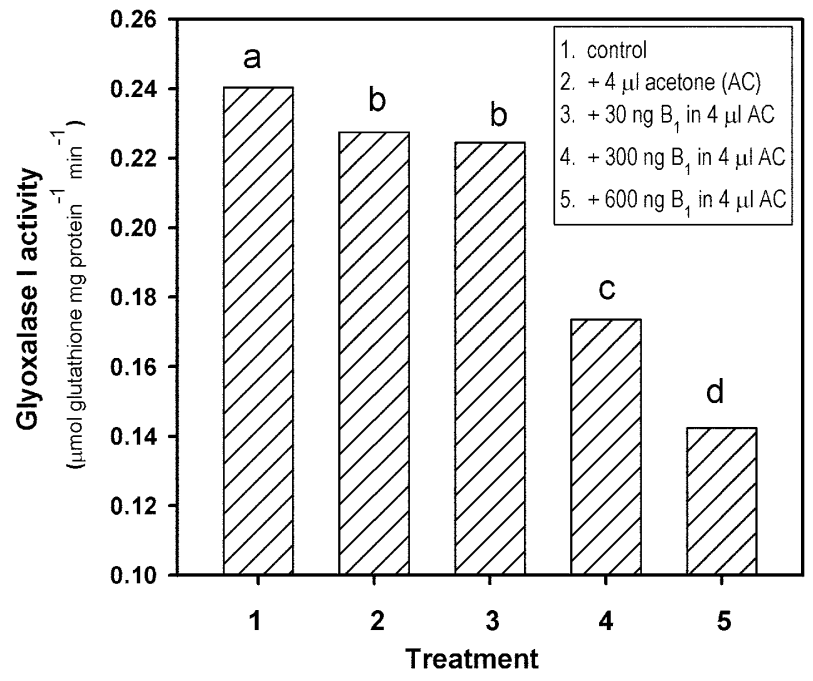

Fig. 6. Effect of aflatoxins on glyoxalase I (GLX-I) activity in vitro. The inset indicates the five treatments under which the GLX-I activities were determined. Treatments 1 and 2 served as controls. Bars labeled with the same letters are not statistically different based on Duncan's multiple range test.

TABLE 2. The effect of fungal infection on glyoxalase I activity and methylglyoxal content in kernels of resistant and susceptible maize genotypes ${ }^{\mathrm{Z}}$

\begin{tabular}{|c|c|c|c|c|c|c|}
\hline \multirow[b]{2}{*}{ Genotypes } & \multicolumn{2}{|c|}{$\begin{array}{c}\text { Mean aflatoxin } \mathrm{B}_{1} \\
\left(\mathrm{ng} \mathrm{g}^{-1} \text { of kernel) }(\mathrm{ppb})\right.\end{array}$} & \multicolumn{2}{|c|}{$\begin{array}{c}\text { GLX-I activity } \\
\left(\mu \mathrm{mol} S \text {-lactoylglutathione } \mathrm{mg}^{-1} \text { protein } \mathrm{min}^{-1}\right)\end{array}$} & \multicolumn{2}{|c|}{$\begin{array}{c}\text { MG content } \\
\left(\mu \mathrm{g} \mathrm{g}^{-1} \text { of kernel }\right)\end{array}$} \\
\hline & Noninfected & Infected & Noninfected & Infected & Noninfected & Infected \\
\hline B73 (S) & ND & $111.85 \mathrm{bc}$ & $0.160 \mathrm{~cd}$ & $0.129 \mathrm{~d}$ & 26.9 ef & $42.2 \mathrm{~cd}$ \\
\hline G4666 (S) & ND & $337.03 \mathrm{a}$ & $0.167 \mathrm{~cd}$ & $0.124 \mathrm{~d}$ & $34.7 \mathrm{def}$ & $85.7 \mathrm{a}$ \\
\hline P3154 (S) & ND & $205.37 \mathrm{ab}$ & $0.172 \mathrm{~cd}$ & $0.146 \mathrm{~d}$ & $23.9 \mathrm{f}$ & 29.0 ef \\
\hline MAS (R) & ND & $77.78 \mathrm{c}$ & $0.195 \mathrm{c}$ & $0.286 \mathrm{a}$ & $48.7 \mathrm{bcd}$ & $51.4 \mathrm{bcd}$ \\
\hline $\operatorname{Mp} 420(\mathrm{R})$ & ND & $99.06 \mathrm{c}$ & $0.270 \mathrm{ab}$ & $0.285 \mathrm{a}$ & $60.3 \mathrm{~b}$ & $57.0 \mathrm{bc}$ \\
\hline T115 (R) & ND & $143.76 \mathrm{bc}$ & $0.294 \mathrm{a}$ & $0.328 \mathrm{a}$ & $46.8 \mathrm{bcd}$ & $42.4 \mathrm{~cd}$ \\
\hline
\end{tabular}

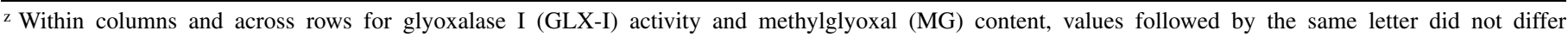
significantly by Duncan's multiple range test. Kernels were inoculated with either sterile water (Noninfected) or an Aspergillus flavus conidia suspension (Infected) and incubated for 7 days before the measurements. $\mathrm{R}=$ resistant, $\mathrm{S}=$ susceptible, and ND = not detectable. 


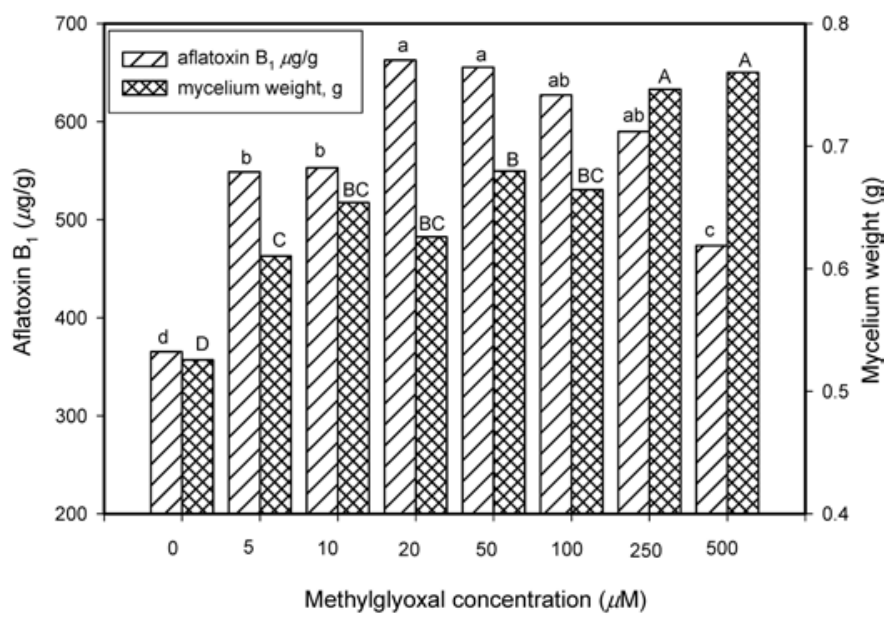

Fig. 7. Effect of methylglyoxal on aflatoxin production and fungal growth of Aspergillus flavus in vitro. Bars labeled with the same letters (case sensitive for the two parameters measured) are not statistically different based on Duncan's multiple range test. Striped and filled bars represent aflatoxin production and fungal mycelium dry weight, respectively, after 4 days of culturing in $\mathrm{A}+\mathrm{M}$ medium containing various concentrations of methylglyoxal. sented here represent combined data from two repeats for all five genes examined in this study, and were normalized to the level of aflR in the control medium. Under normal growth conditions without MG (control medium), the transcript levels for aflR and nor 1 in control medium were similar, and that of aflJ was approximately half of aflR. The expression of the other two genes, ver 1 and $p k s$, was $\approx 25$-fold higher than aflR. When the cultures were treated with low concentrations of MG $(5$ or $20 \mu \mathrm{M})$, three different expression patterns were observed depending on the genes. The first pattern was seen for aflR and nor 1 , in which the expression of both genes increased significantly (78 and 30\%, respectively) as the concentration of MG in the medium was increased from 0 to $20 \mu \mathrm{M}$ (Fig. 8A and C). The second pattern was seen for aflJ and ver 1, in which the gene expression did not change as MG concentration increased from 0 to $20 \mu \mathrm{M}$ in the cultures (Fig. 8B and D). The expression of pks, which decreased as MG concentration increased in the cultures, represents the third pattern (Fig. 8E). As the concentration of MG in the medium continued to increase to 50 or $250 \mu \mathrm{M}$, a significant decrease in the level of expression of all the aflatoxin biosynthesis genes examined in this study compared with the untreated control was observed. The expression of nor 1 and aflJ decreased to approximately one-half and one-sixth, respectively, of the untreated control in the presence of $250 \mu \mathrm{M}$ MG. This treatment also de-
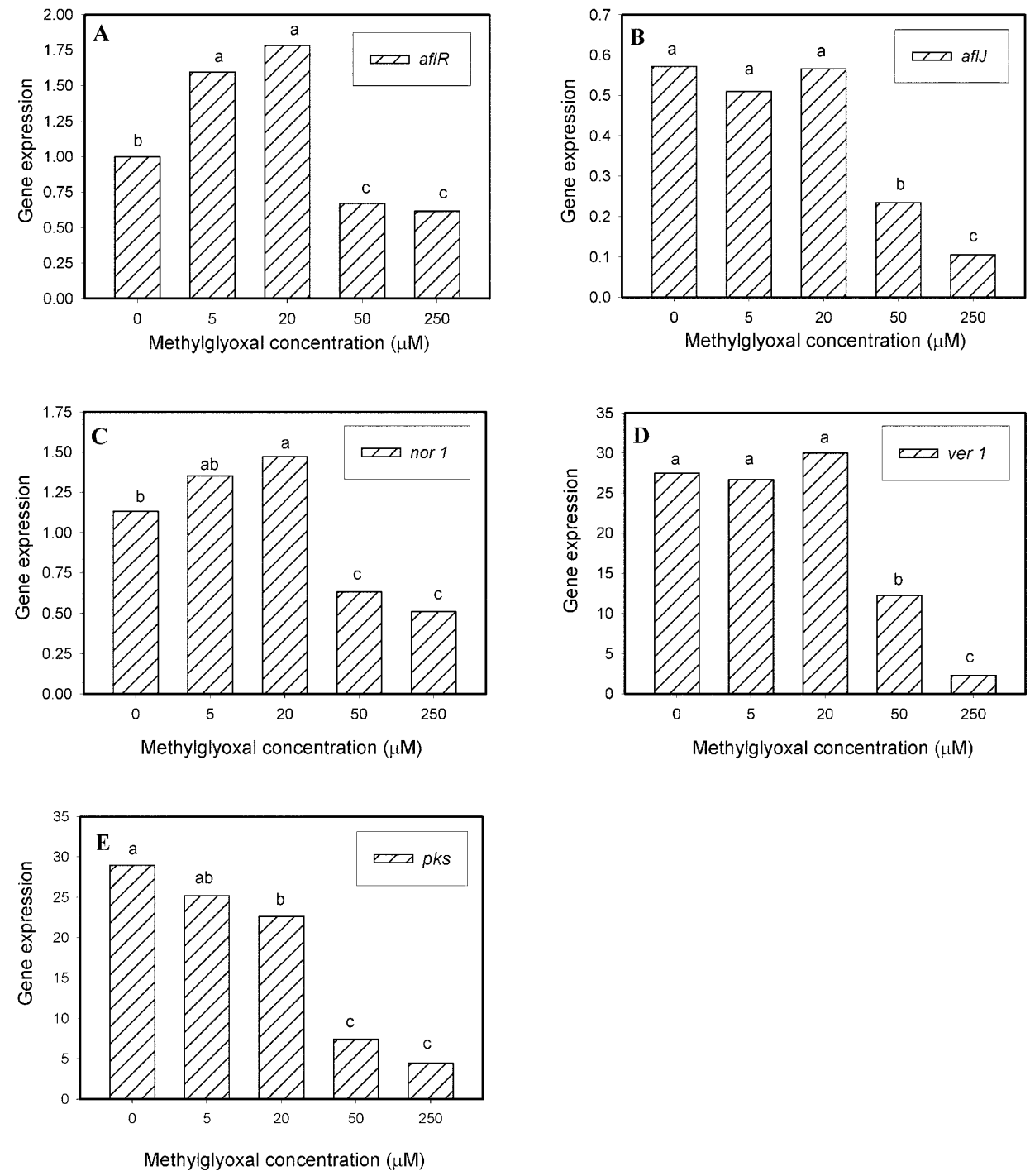

Fig. 8. Expression of aflatoxin biosynthetic pathway genes in Aspergillus flavus when grown in $\mathrm{A}+\mathrm{M}$ medium containing various concentrations of methylglyoxal. Transcript levels of five aflatoxin biosynthesis pathway genes were measured using realtime polymerase chain reaction and first were normalized to $18 \mathrm{~S}$ ribosomal RNA (internal normalizer). The mean level (fold) of expression shown here is relative to that of aflR in the control medium, which is defined as 1. 
creased the level of ver 1 and pks transcripts, two of the highly expressed genes, by 11.8- and 6.5-fold, respectively, compared with the control (Fig. 8).

\section{DISCUSSION}

A. flavus infection of maize kernels occurs early during kernel development. However, aflatoxin accumulation does not occur until late in the maturation phase, coinciding with the onset of dehydration (31). Increased temperatures and drought, which often occur together, are major factors known to enhance aflatoxin contamination of maize kernels (31). Recent studies have found higher levels of stress-related proteins and highly hydrophilic storage proteins in kernels of resistant genotypes compared with susceptible genotypes (13). This may enable resistant kernels to effectively induce an active defense response upon fungal attack, even under stresses caused by heat or drought.

In the present study, a GLX-I protein, expressed at higher levels in resistant maize kernel embryos than in susceptible ones (13), was identified on the basis of peptide sequence analysis. Here, we also report the cloning of a $g l x-I$ gene from maize for the first time. Sequence homology comparisons indicate that maize GLX-I belongs to the long-type glyoxalase family (280 to 295 amino acids), which contains two highly homologous domains. It is believed that the monomeric two-domain long-type GLX-I genes are evolved from dimeric one-domain short-type GLX-I genes through the process of gene duplication and threedimensional domain swapping (10).

GLX-I is present in many organisms, such as fungi, plants, and animals (36), but its role has not been well investigated in plants. It catalyzes the conversion of MG, a potent cytotoxic compound, to nontoxic D-lactate in the presence of glutathione and GLX-II (26). MG is known to arrest growth and react with DNA and protein and increase sister chromatid exchanges $(21,30,35)$. MG is produced spontaneously in all organisms at low micromolar concentrations under physiological conditions from glycolysis and photosynthesis intermediates, glyceraldehyde-3-phosphate and dihydroxyacetone phosphate $(20,25)$. It also can be produced enzymatically by triose phosphate isomerase or by MG synthetase $(20,25)$. Recent studies found that, in addition to dehydrin and group 3 late embryogenesis abundant proteins, $g l x-I$ also was induced during drought stress (5) in Sporobolus stapfianus, and in response to salt and water stresses in B. juncea (36) and tomato (20), suggesting an important role for GLX-I in conferring tolerance to plants under those stress conditions (36).

To investigate the possibility of a direct relationship between GLX-I and maize kernel aflatoxin resistance, levels of GLX-I activity in dry, noninfected (imbibed), and infected kernels of resistant and susceptible lines were examined. It was determined that resistant lines generally have higher constitutive levels of GLX-I activity than susceptible ones. The exceptions to this are the low GLX-I activity in the resistant line MI82 and high GLX-I activity in susceptible genotype Va35, suggesting that, in addition to GLX-I, other factors also may play an important role in kernel resistance (15). Trypsin inhibitor proteins (14), chitinases (28), and kernel phenolic compounds (22) are other compounds that may contribute as well. Recent genetic studies have shown that resistance to A. flavus infection and aflatoxin production in maize is a multiple-gene-controlled trait (or a quantitative trait) $(17,37)$. The higher GLX-I activity in noninfected (imbibed) kernels (Table 2) compared with dry kernels (Fig. 5) indicated that imbibition may induce GLX-I activity in both resistant and susceptible genotypes. However, fungal infection did not appear to have a clear effect on the GLX-I activity. Only one resistant genotype (MAS) showed a significant increase in GLX-I activity due to infection. This observed increase of GLX-I activity after infection was not likely contributed by A. flavus, because the same was not observed in susceptible genotypes where more colonization usually is observed
(8). Instead, this increase likely is due to the induction of $g l x-I$ expression in response to A. flavus infection (12). In B. juncea, an increase in $g l x-I$ expression and GLX-I activity has been reported when the seedlings were subjected to salt stress (36).

After fungal infection, the level of MG did not increase in resistant genotypes. This lack of increase could be due to the relatively higher levels of GLX-I activity observed in resistant infected kernels. However, two of three susceptible genotypes showed a significant increase in MG content in infected kernels. Increases in $\mathrm{MG}$ production when cells are under stress also has been reported (25). An elevation in MG content in susceptible genotypes, combined with low GLX-I activities, could weaken the kernel's ability to defend against fungal infection. In addition, the present study also demonstrated that the presence of aflatoxin at levels of 300 to $600 \mathrm{ppb}$ significantly inhibited maize GLX-I activity in vitro. A similar inhibitory effect of GLX-I activity by aflatoxin also has been reported in mice $(3,4)$. Therefore, the accumulation of high levels of aflatoxin in susceptible kernels after infection, usually ranging from hundreds to thousands of parts per billion or even higher (31), could further inhibit the kernel GLX-I activity.

The current study also examined possible adverse effects of an increase in MG concentration on A. flavus. No in vitro evidence of MG inhibition of fungal growth was observed. However, an unexpected induction of fungal growth and aflatoxin production at low MG concentrations ( 5 to $20 \mu \mathrm{M}$ ) was observed. An examination of aflatoxin biosynthetic pathway gene expression indicated that the pathway regulatory gene afl R transcript was significantly upregulated $(78 \%)$ when the culture was treated with $\mathrm{MG}$ at $20 \mu \mathrm{M}$ for 4 days. Other pathway genes, such as nor 1 , also showed an increase in transcript levels under the same conditions.

The present study suggests an important role for GLX-I in resistance through the control of MG levels and, therefore, aflatoxin induction, in A. flavus infected kernels. However, other factors also may contribute to kernel resistance. Whether or not GLX-I plays another role in kernels related to stress tolerance requires further investigation. Strategies such as gene silencing using RNAi (38) or quantitative trait loci mapping of $g l x-I$ in the future could provide the basis for gaining greater clarity as to the significance of GLX-I in host resistance or stress tolerance.

\section{ACKNOWLEDGMENTS}

This study was supported by United States Department of Agriculture (USDA) cooperative agreement 58-6435-2-130, and USDA National Research Initiative Competitive Grant 2002-35201-12541. We thank C. Ambrogio, D. Hadrick, and J. Veal for technical assistance; P.-K. Chang for providing real-time PCR primers; and H.-J. Kim, B. B. Rees, and J. Yu for critical reviewing of the manuscript.

\section{LITERATURE CITED}

1. Adye, J., and Mateles, R. I. 1964. Incorporation of labeled compounds into aflatoxin. Biochim. Biophys. Acta 86:418-420.

2. Altschul, S. F., Madden, T. L., Schäffer, A. A., Zhang, J.-H., Zhang, Z., Miller, W., and Lipman, D. J. 1997. Gapped BLAST and PSI-BLAST: A new generation of protein database search programs. Nucleic Acids Res. 25:3389-3402.

3. Ankrah, N. A. 1995. Alteration of glucose tolerance in mice fed low levels of aflatoxins and with depressed glyoxalase-I activity. Vet. Hum. Toxicol. 37:59-61.

4. Ankrah, N. A., Ekuban, F. A., and Asamoah, E. B. 1990. Depressant effect of very low levels of aflatoxin $B_{1}$ on mouse glyoxylase-I activity and methylglyoxal disposal. Biochem. Pharmacol. 39:1261-1263.

5. Blomstedt, C. K., Gianello, R. D., Hamill, J. D., Neale, A. D., and Gaff, D. F. 1998. Drought-stimulated genes correlated with dessication tolerance of the resurrection grass Sporobolus stapfianus. Plant Growth Regul. 24:153-161.

6. Bradford, M. M. 1976. A rapid and sensitive method for the quantitation of microgram quantities of protein utilizing the principle of protein-dye binding. Anal. Biochem. 72:248-254. 
7. Brown, R. L., Chen, Z.-Y., Cleveland, T. E., and Russin, J. S. 1999. Advances in the development of host resistance to aflatoxin contamination by Aspergillus flavus. Phytopathology 89:113-117.

8. Brown, R. L., Cleveland, T. E., Payne, G. A., Woloshuk, C. P., Campbell, K. W., and White, D. G. 1995. Determination of resistance to aflatoxin production in maize kernels and detection of fungal colonization using an Aspergillus flavus transformant expressing Escherichia coli $\beta$-glucuronidase. Phytopathology 85:983-989.

9. Brown, R. L., Cotty, P. J., Cleveland, T. E., and Widstrom, N. W. 1993. Living maize embryo influences accumulation of aflatoxin in maize kernels. J. Food Prot. 56:967-971.

10. Cameron, A. D., Olin, B., Ridderstrom, M., Mmannervik, B., and Jones, T. A. 1997. Crystal structure of human glyoxalase-I-Evidence for gene duplication and 2D domain swapping. EMBO J. 16:3386-3395.

11. Campbell, K. W., and White, D. G. 1995. Evaluation of corn genotypes for resistance to Aspergillus ear rot, kernel infection, and aflatoxin production. Plant Dis. 79:1039-1045.

12. Chen, Z.-Y., Brown, R. L., Cleveland, T. E., Damann, K. E., and Russin, J. S. 2001. Comparison of constitutive and inducible maize kernel proteins of genotypes resistant or susceptible to aflatoxin production. J. Food Prot. 64:1785-1792.

13. Chen, Z.-Y., Brown, R. L., Damann, K. E., and Cleveland, T. E. 2002. Identification of unique or elevated levels of kernel proteins in aflatoxinresistant maize genotypes through proteome analysis. Phytopathology 92:1084-1094.

14. Chen, Z.-Y., Brown, R. L., Lax, A. R., Guo, B. Z., Cleveland, T. E., and Russin, J. S. 1998. Resistance to Aspergillus flavus in corn kernels is associated with a 14-kDa protein. Phytopathology 88:276-281.

15. Chen, Z.-Y., Cleveland, T. E., Brown, R. L., Bhatnagar, D., Cary, J. W., and Rajasekaran, K. 2002. Corn as a source of antifungal genes for genetic engineering of crops for resistance to aflatoxin contamination. Crop Biotechnol. ACS Symp. Ser. 829:131-150.

16. Cleveland, T. E., Bhatnagar, D., and Brown, R. L. 1991. Aflatoxin production via cross-feeding of pathway intermediates during cofermentation of aflatoxin pathway-blocked Aspergillus parasiticus mutants. Appl. Environ. Microbiol. 57:2907-2911.

17. Davis, G. L., and Williams, W. P. 1999. QTL for aflatoxin reduction in maize. Maize Genet. Conf. 41:22.

18. Deswal, R., and Sopory, S. K. 1999. Glyoxalase I from Brassica juncea is a calmodulin stimulated protein. Biochim. Biophys. Acta 1450:460467.

19. Diener, U. L., Cole, R. J., Sanders, T. H., Payne, G. A., Lee, L. S., and Klich, M. A. 1987. Epidemiology of aflatoxin formation by Aspergillus flavus. Annu. Rev. Phytopathol. 25:249-270.

20. Espartero, J., Sanchez-Aguayo, I., and Pardo, J. M. 1996. Molecular characterization of glyoxalase-I from a higher plant; Upregulation by stress. Plant Mol. Biol. 29:1223-1233.

21. Figgin, P., Bassi, A. M., Finollo, R., and Brambilla, G. 1985. Induction of sister chromatid exchanges in Chinese hamster ovary cells by the biotic ketoaldehyde methylglyoxal. Mutat. Res. 144:189-191.

22. Gembeh, S. V., Brown, R. L., Grimm, C., and Cleveland, T. E. 2001. Identification of chemical components of corn kernel pericarp wax associated with resistance to Aspergillus flavus infection and aflatoxin production. J. Agric. Food Chem. 49:4635-4641.
23. Guo, B. Z., Russin, J. S., Cleveland, T. E., Brown, R. L., and Widstrom, N. W. 1996. Resistance to aflatoxin contamination in corn as influenced by relative humidity and kernel germination. J. Food Prot. 59:276-281.

24. Hsieh, D. P. H. 1989. Potential human health hazards of mycotoxins. Pages 69-80 in: Mycotoxins and Phycotoxins. S. Natori, K. Hashimoto, and Y. Ueno, eds. Elsevier, Amsterdam.

25. Inoue, Y., Tsujimoto, Y., and Kimura, A. 1998. Expression of the glyoxalase I gene of Saccharomyces cerevisiae is regulated by high osmolarity glycerol mitogen-activated protein kinase pathway in osmotic stress response. J. Biol. Chem. 273:2977-2983.

26. Johansen, K. S., Svendsen, I. I., and Rasmussen, S. K. 2000. Purification and cloning of the two domain glyoxalase I from wheat bran. Plant Sci. 155:11-20.

27. Lozovaya, V. V., Waranyuwat, A., and Widholm, J. M. 1998. $\beta-1,3-G l u-$ canase and resistance to Aspergillus flavus infection in maize. Crop Sci. 38:1255-1260.

28. Moore, K. G., Price, M. S., Boston, R. S., Weissinger, A. K., and Payne, G. A. 2004. A chitinase from Tex6 maize kernels inhibits growth of Aspergillus flavus. Phytopathology 94:82-87.

29. Nichols, T. E., Jr. 1983. Economic impact of aflatoxin in corn. South. Coop. Ser. Bull. 279:67-71.

30. Papoulis, A., Al-Abed, Y., and Bucala, R. 1995. Identification of N2-(-1carboxylethyl) guanine (CEG) as a guanine advanced glycosylation endproduct. Biochemistry 34:648-655.

31. Payne, G. A. 1998. Process of contamination by aflatoxin-producing fungi and their impact on crops. Pages 279-306 in: Mycotoxins in Agriculture and Food Safety. K. K. Sinha and D. Bhatnagar, eds. Marcel Dekker, New York.

32. Racker, E. 1951. The mechanism of action of glyoxalases. J. Biol. Chem. 190:685-696.

33. Scott, G. E., and Zummo, N. 1988. Sources of resistance in maize to kernel infection by Aspergillus flavus in the field. Crop Sci. 28:504-507.

34. Swegel, M., Kramer, K. J., and Muthukrishnan, S. 1992. Properties of barley seed chitinases and release of embryo-associated isoforms during early stages of imbibition. Plant Physiol. 99:1009-1014.

35. Thornalley, P. J. 1990. The glyoxalase system: New developments towards functional characterization of metabolic pathways fundamental to biological life. Biochem. J. 269:1-11.

36. Veena, Reddy, V. S., and Sopory, S. K. 1999. Glyoxalase I from Brassica juncea: Molecular cloning, regulation and its over-expression confer tolerance in transgenic tobacco under stress. Plant J. 17:385-395.

37. Walker, R. D., and White, D. G. 2001. Inheritance of resistance to Aspergillus ear rot and aflatoxin production of corn from CI2. Plant Dis. 85:322-327.

38. Wesley, S. V., Helliwell, C. A., Smith, N. A., Wang, M. B., Rouse, D. T., Liu, Q., Gooding, P. S., Singh, S. P., Abbott, D., Stoutjesdijk, P. A., Robinson, S. P., Gleave, A. P., Green, A. G., and Waterhouse, P. M. 2001. Construct design for efficient, effective and high-throughput gene silencing in plants. Plant J. 27:581-590.

39. Widstrom, N. W., McMillian, W. W., and Wilson, D. M. 1987. Segregation for resistance to aflatoxin contamination among seeds on an ear of hybrid maize. Crop Sci. 27:961-963.

40. Yu, J. J., Bhatnagar, D., and Ehrlich, K. C. 2002. Aflatoxin biosynthesis. Rev. Iberoam. Micol. 19:191-200. 